\title{
High Temperature Superconducting Fault Current Limiters as Enabling Technology in Electrical Grids with Increased Distributed Generation Penetration
}

\author{
João Murta Pina ${ }^{1}$, Mário Ventim Neves ${ }^{1}$, Alfredo Álvarez ${ }^{2}$, \\ and Amadeu Leão Rodrigues ${ }^{1}$ \\ ${ }^{1}$ Centre of Technology and Systems, \\ Faculdade de Ciências e Tecnologia, Nova University of Lisbon \\ Monte de Caparica, 2829-516 Caparica, Portugal \\ jmmp@fct.unl.pt, ventim@uninova.pt, leao@uninova.pt \\ 2 "Benito Mahedero" Group of Electrical Applications of Superconductors, \\ Escuela de Ingenierías Industriales, University of Extremadura \\ Avenida de Elvas s/n, 06006 Badajoz, Spain \\ aalvarez@unex.es
}

\begin{abstract}
Amongst applications of high temperature superconductors, fault current limiters are foreseen as one of the most promising in power systems. Several topologies have been developed in the last years, taking advantage of different superconductors' properties. Increasing distributed generation (DG) penetration, based on renewable energy, adds new short-circuit sources to electrical grids, which brings several energy quality and protection issues. Superconducting fault current limiters can obviate these problems, representing thus an enabling technology for DG penetration. In this paper current limiter topologies are presented, its operations principles, strengths and weaknesses, in the context of these DG grids. In the end, future trends are discussed.
\end{abstract}

Keywords: Superconducting fault current limiters, distributed generation.

\section{Introduction}

In the past years, several reasons justified the increasing penetration of dispersed, embedded or distributed generation (DG) in electrical grids. Amongst these highlights common demand on greenhouse emissions reduction by the use of renewable energy sources; energy efficiency and rational energy use; diversification of energy sources [1]; availability, short construction times and cheap small generator plants; and the advantages of placing generators near consumers decreasing transmission costs [2]. Nevertheless these favorable aspects, DG still raises several relevant technical questions [3], [4], as network voltage changes (e.g. in partially loaded grids with high renewable generation); power quality issues (e.g. harmonics due to static converters); and conflicts with network protections. The latter relates with increase in short-circuit current levels and grid switchgears' selectivity issues, amongst others.

Restrictions arising from protections conflicts usually prevent additional DG penetration [5]. Adding new rotating generators (e.g. wind turbines) may increase dramatically 
fault levels, as resulting short-circuit currents consist on phasor sum of original fault currents and new DG generators' fault currents [5]. The latter are not considered when grids protections are originally designed. Simulations found in the literature [3], [6], demonstrate that adding DG generators increases considerably fault levels which can exceed the current interrupting capacity of the existing switchgears.

An obvious solution to the above problem is to upgrade grid switchgears, adapting their rating to the new levels, but this may turn economically unfeasible. Reducing new generators fault contributions may be achieved by introducing impedances (transformers or reactors) between these and the corresponding grid, which increases losses. Explosive fuses may also be used but these must be replaced after a fault. In recent years, new technologies appeared, as solid state fault current limiters [7], which have high semiconductors losses and are costly, or high temperature superconducting fault current limiters (SFCL). The latter are detailed in the paper.

Next section addresses authors' contribution to technological innovation. High temperature superconducting (HTS) materials are introduced and SFCL classified according to its characteristics and operation principle. Resistive and inductive types are explained in later sections. Future trends and conclusions are drawn in the end.

\section{Contributions to Technological Innovation}

The vision of future electric grids envisages coexistence of distributed and central generation, energy storage and bidirectional energy flow, between grid and consumers/ producers. SFCL are a promising technology in such environment, addressing issues as safety or power quality. It is thus relevant to develop analysis tools for their dynamic behavior, and authors built such a straightforward algorithm for one type of those limiters (inductive), based on the individual analysis of device's elements (HTS and iron core) [8]. It builds FCL's hysteresis cycle, allowing simulating grids' currents and correctly dimensioning switchgears or transformers.

\section{High Temperature Superconducting Fault Current Limiters}

Superconducting materials are natural fault current limiters, often recognized as one of its most promising power application. In superconducting state they carry high current densities (up to $10^{4} \mathrm{~A} / \mathrm{mm}^{2}$ ) with negligible losses. Under a fault, current density increases. The material loses superconductivity and becomes resistive, limiting current. Once fault suppressed, it returns naturally to the superconducting state. This is the principle of the resistive SFCL, detailed in Section 3. These devices use material non linear resistivity in superconducting/normal state transition.

\subsection{High Temperature Superconducting Materials}

The main macroscopic properties of the first discovered superconducting materials (superconductivity was discovered by the Dutch physicist Kamerlingh Onnes in mercury, in 1911) were the abrupt loss of electrical resistivity under a certain critical temperature and the exclusion of magnetic flux from the material (Meissner effect). For decades, superconductors' highest critical temperature was around $20 \mathrm{~K}$, so they 
had to be cooled with liquid helium (boiling temperature $4.22 \mathrm{~K}$ ) in expensive cryogenic systems. The discovery of superconductivity in ceramic copper oxides, as $\mathrm{YBa}_{2} \mathrm{Cu}_{3} \mathrm{O}_{x}$, boosted this temperature to above liquid nitrogen boiling temperature, 77 $\mathrm{K}$, reducing dramatically cryogenics' cost. In these high temperature superconductors, magnetic flux is not completely expelled from material's core, rather penetrates it in quantized amounts known as fluxoids in the so called mixed state. The transition from superconducting to normal state, known as quench, depends on the values of current density, temperature and flux density in the material.

\subsection{Classification of Superconducting Fault Current Limiters}

SFCL may be classified according to the way they are introduced in the grid, their use of quench, or employment of iron cores, amongst others.

Single-phase SFCLs' classification is presented in Fig. 1. It depends on how the devices are inserted in the grid: in series (resistive), magnetically linked (inductive) or through a rectifier bridge (rectifier). In this paper resistive and inductive topologies are presented. The rectifier SFCL was abandoned due to high voltage failures and high losses on the semiconductor elements used.

Three-phase SFCLs are the most important devices in power applications. They are built by three single-phase modules or using an exclusive three-phase design [9]. Although the latter approach uses less HTS material than three single-phase SFCL modules, a fault in one phase affects the remaining ones and this device is restricted to symmetric loads (e.g. motors). Thus, this approach is not presented in the paper.

\section{Resistive Type Fault Current Limiter}

The resistive SFCL is represented in Fig. 2. It is built, in its most basic form, by an HTS element in series with the grid, with a shunt resistance, $R_{\mathrm{s}}$, described later.

\subsection{Operation Principle}

In normal grid operation, the SFCL has negligible impedance and associated losses, being electrically invisible to the grid [10]. Under a fault, the current exceeds HTS's critical value, leading to quench. HTS's impedance rises abruptly, limiting current.

Reference [11] proposes a simple qualitative explanation for the resistive SFCL behavior under a fault, based on the analysis of the curve that relates electrical field, $E$, with current density, $J$, in HTS materials, see Fig. 3 and Fig. 4:

- If HTS length is "high", $E$ is "low" so as $J$ and ohmic losses. The heat produced is dissipated by the cryogenic system and material's temperature remains constant. Although this represents the ideal behavior of the SFCL, the amount of bulk HTS used (up to hundreds of meters [12]) may turn it unfeasible.

- If HTS length is "low", $E$ and $J$ are "high". Thus, the initial current peak may not be sufficiently limited. Losses are "high" and HTS fast heating may not be absorbed by cryogenics, which can lead to material's collapse.

- In a "moderate" length HTS, E, $J$ and losses are also "moderate". In this case there is an HTS slow heating. 
The shunt resistance $R_{\mathrm{s}}$ protects HTS material against hot spots formation, i.e. regions that enter normal state, increasing locally their resistivity and temperature and degrading the material. $R_{\mathrm{s}}$ provides an alternative low resistance path for current.

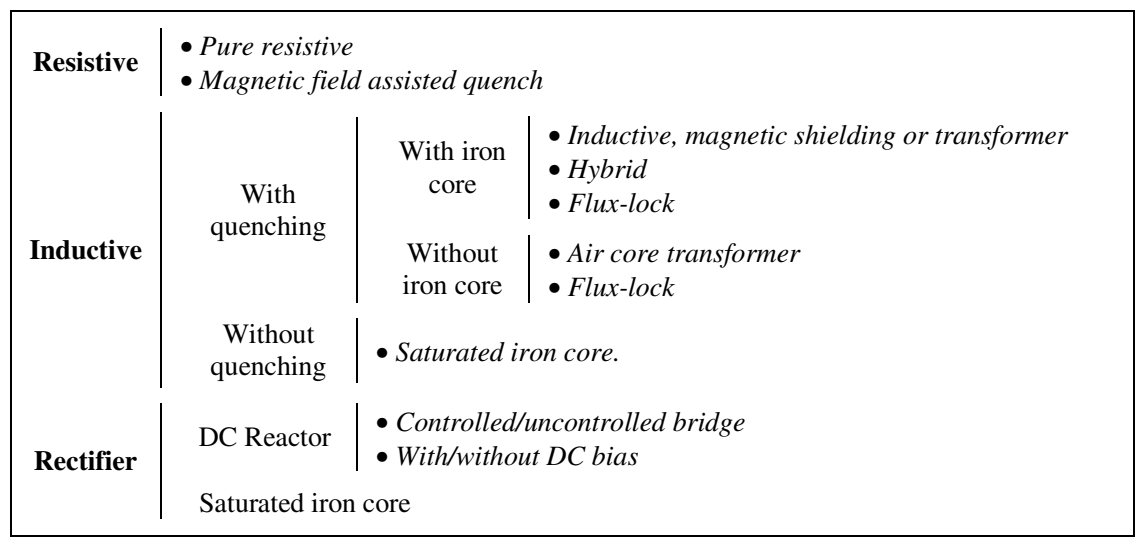

Fig. 1. Classification of single-phase superconducting fault current limiters

\subsection{Resistive SFCL Demonstrators}

Under the framework of CURL 10 project [13] a three-phase $10 \mathrm{kV} / 10 \mathrm{MVA}$ was build with superconducting bifilar coils as limiting elements. The system operated in field conditions in RWE company's electric grid in Netphen, Germany, coupling two $10 \mathrm{kV}$ buses. Yet, no fault occurred in that period. This concept evolved to magnetic field assisted quench SFCL, referred in the last section.

A limiter using thin film HTS was successfully tested in a $14 \mathrm{kV}$ single-phase grid [14]. $10 \mathrm{kA}$ prospective current is limited to $445 \mathrm{~A}$ in the first peak and previously to less than 140 A. Although operation is demonstrated, thin films' high cost, namely for transmission grids with large number of elements, lead to abandoning this approach.

The New Energy and Industrial Technology Development Organization, NEDO, is developing a resistive limiter based on HTS coated conductors. In a $3.8 \mathrm{kV}$ singlephase grid, a $17 \mathrm{kA}$ prospective current is limited to $700 \mathrm{~A}$ [15].

\subsection{Conclusions}

Resistive SFCL allows fast limiting action, in less than half cycle, since HTS element is in series with the grid. It is also a compact device. Yet, several elements must be series connected in order to achieve desired limiting performance, degrading quench homogeneity which can lead to material's destruction. Current leads are also needed, to make current transition from room to cryogenic environment. Recovery time is usually in the order of a few seconds [11]. Devices demonstrated their technical viability in medium voltage distribution networks, usually associated with DG. 


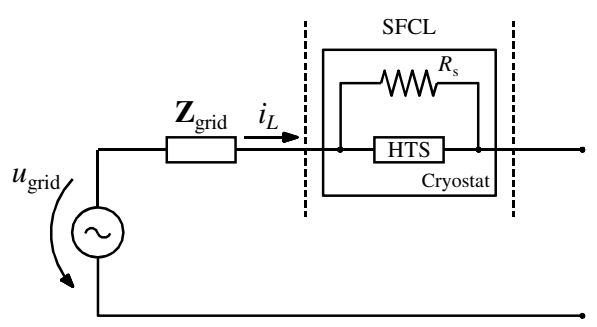

Fig. 2. Resistive superconducting fault current limiter electrical diagram

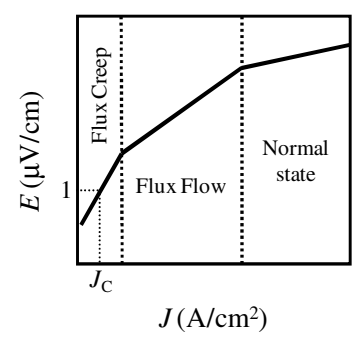

Fig. 3. HTS $E-J$ qualitative dependence. The $1 \mu \mathrm{V} / \mathrm{cm}$ criterion defines critical current density, $J_{c}$, below which the material is in the superconducting state. Above $J_{c}$ and below normal state, the material is in the mixed state.

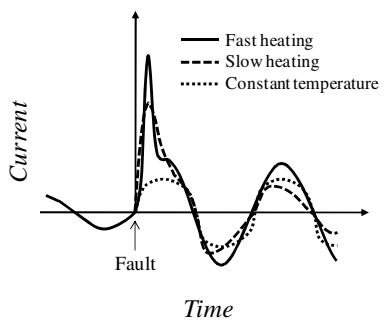

Fig. 4. Different types of response under fault in resistive SFCL with distinct lengths (adapted from [11])

\section{Inductive Type Fault Current Limiter}

The inductive type SFCL, introduced in [16], is magnetically linked with the power line, behaving as a current transformer with short-circuited secondary, see Fig. 5. The primary is fed by the power line, while the secondary is built by an HTS element, usually a cylinder or a stack of cylinders. The primary and secondary concentrically embrace an iron core forming a closed or open path for magnetic flux.

\subsection{Operation Principle}

In normal operation, the primary's magnetic flux induces currents in the HTS that shield magnetically the iron core, leading to a negligible primary's linked flux (no flux lines penetrate the core) and consequently to negligible impedance (only related to leakage), see Fig. 6. Under a fault, line current increases abruptly and the HTS is no longer able to shield flux. It finds a preferential path through iron where it is amplified, see Fig. 7. Primary's linked flux increases by some orders of magnitude, increasing inductance and limiting current. Current evolution is represented in Fig. 8. Limitation is visible in the first half cycle, although it gets more effective after that. 


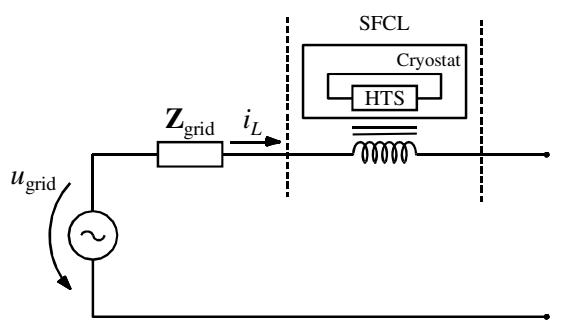

Fig. 5. Inductive superconducting fault current limiter electrical diagram

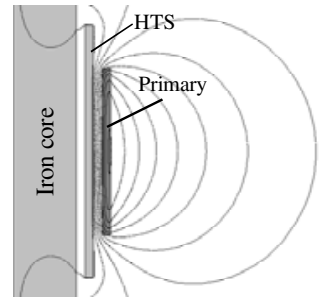

Fig. 6. Axisymmetric representation of an inductive SFCL in normal operation. Primary's flux is shielded from iron core by HTS induced currents.

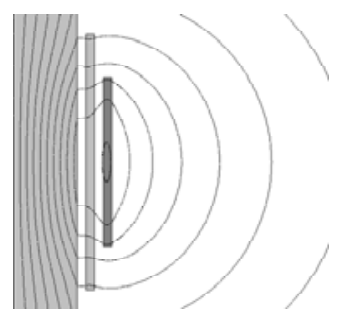

Fig. 7. SFCL under a fault. Flux penetrates iron core, increasing device's impedance.

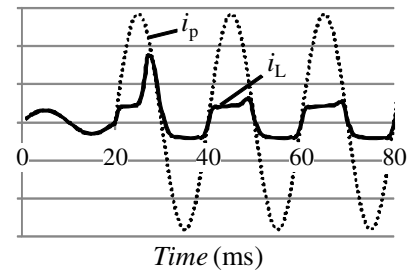

Fig. 8. Evolution of current in the circuit, $i_{\mathrm{L}}$, under a fault, applied at $20 \mathrm{~ms}$. Prospective current, $i_{\mathrm{p}}$, is also shown. Results are taken from [8].

\subsection{Inductive SFCL Demonstrators}

The most relevant demonstrator found in the literature was implemented by $\mathrm{ABB}$ Company, namely a three-phase $10.5 \mathrm{kV} / 1.2$ MVA built in a three leg core [17]. In laboratory, $60 \mathrm{kA}$ prospective currents were limited to $700 \mathrm{~A}$ in the first half cycle and to less than $250 \mathrm{~A}$ after $50 \mathrm{~ms}$. After 6 months of operation in Kraftwerk am Löentsch hydroelectric plant, Swiss, the device "...did not face any major problems". The re-cooling time for the material was in the order of two to ten seconds. 


\subsection{Conclusions}

The inductive SFCL has some important advantages compared with the resistive one. Current leads, source of ohmic losses, are dispensable, limiting impedance is easily adjusted through primary turns, and hot spots are easily suppressed by cryogenics. Yet, it has considerable weight and volume due to iron cores. Large bulk superconductors are also hard to produce in good homogeneity conditions. Nevertheless, technical viability in distribution networks is demonstrated.

\section{Future Trends and Conclusions}

Resistive SFCL are object of great research due to their compactness. Yet, hot spot formation is a major issue, as it might irreversibly damage HTS elements. A novel design called magnetic field assisted quench is being developed in the framework of CULT 110 project [18], using applied field's quench dependence. HTS element is placed inside a conventional coil and these are shunted. In normal operation, current flows through the "zero" impedance element. Under a fault, a voltage is developed at its terminals. Part of the current deviates to the coil, originating magnetic flux that forces homogeneous and fast quench, avoiding hot spots.

Although less compact, robustness of inductive SFCL makes them attractive in distribution networks, where DG is applied. The algorithm developed by the authors [8] is thus of utmost relevance.

SFCL limit DG short-circuit currents, allowing its increasing penetration. Although cryogenics is still the most relevant technical challenge in superconductivity, it can be diluted if other HTS technologies are present, as lossless superconducting transformers, flywheels (energy is stored in a rotating mass, using HTS bearings to avoid contact) and SMES (superconducting magnetic energy storage, energy is stored in the magnetic field of an HTS coil). The last ones are related with energy storage and power quality, key issue in the advent of future electric grids, relying on DG, energy storage and bidirectional energy flow and complying with strict standards.

\section{References}

1. CIGRE Sudy Committee 37-23: Impact of increasing contributions of dispersed generation on the power systems. Technical report, CIGRE (1999)

2. CIRED Working Group 04: Dispersed generation. Preliminary report, CIRED Conference, Nice, France (1999)

3. Conti, S.: Analysis of distribution network protection issues in presence of dispersed generation. Electrical Power Systems Research 79, 49-56 (2009)

4. Jenkins, N., Allan, R., Crossley, P., Kirschen, D., Strbac, G.: Embedded Generation. The Institution of Engineering and Technology, London (2000)

5. Boutsika, T., Papathanassiou, S.: Short-circuit calculations in networks with distributed generation. Electrical Power Systems Research 78, 1181-1191 (2008)

6. Tran-Quoc, T., Andrieu, C., Hadjsaid, N.: Technical impacts of small distributed generation units on LV networks. IEEE Power Engineering Society General Meeting 4, 2459 2464 (2003) 
7. Kunde, K., Kleimaier, M., Klingbeil, L.: Integration of fast acting electronic fault current limiters (EFCL) in medium voltage systems. In: CIRED 17th International Conference on Electricity Distribution, Barcelona, Spain (2003)

8. Pina, J., Suárez, P., Ventim Neves, M., Álvarez, A., Leão Rodrigues, A.: Reverse Engineering of Inductive Fault Current Limiters. Accepted for publication. In: 9th European Conference on Applied Superconductivity, EUCAS 2009, Dresden, Germany (2009)

9. Sato, T., Yamaguchi, M., Fukui, S., Watanabe, Y., Matsumura, T., Shimizu, H.: A Study on DC S/N Transition Type Superconducting Fault Current Limiting Interrupter. IEEE Trans. App. Superconductivity 13, 2088-2091 (2003)

10. Noe, M., Juengst, K.-P., Werfel, F., Cowey, L., Wolf, A., Elschner, S.: Investigation of high-Tc bulk material for its use in resistive superconducting fault current limiters. IEEE Trans. App. Superconductivity 11, 1960-1963 (2001)

11. Paul, W., Chen, M., Lakner, M., Rhyner, J., Braun, D., Lanz, W.: Fault current limiter based on high temperature superconductors - different concepts, test results, simulations, applications. Physica C 354, 27-33 (2001)

12. Shimizu, H., Yokomizu, Y., Matsumura, T., Murayama, N.: Proposal of Flux Flow Resistance Type Fault Current Limiter using Bi2223 High Tc Superconducting Bulk. IEEE Trans. App. Superconductivity 12, 876-879 (2002)

13. Bock, J., Breuer, F., Walter, H., Elschner, S., Kleimaier, M., Kreutz, R., Noe, M.: CURL 10: Development and Field-Test of a 10 kV/10 MVA Resistive Current Limiter Based on Bulk MCP-BSCCO 2212. IEEE Trans. App. Superconductivity 15, 1955-1960 (2005)

14. Sim, J., Park, K., Kim, H., Kang, J., Lee, B., Kim, H., Oh, I., Hyun, O.: 14 kV single-phase superconducting fault current limiter based on YBCO films. Cryogenics 47, 183-188 (2007)

15. Yazawa, T., Koyanagi, K., Takahashi, M., Ono, M., Toba, K., Takigami, H., Urata, M., Iijima, Y., Saito, T., Ameniya, N., Shiohara, Y.: Superconducting fault current limiter using high-resistive YBCO tapes. Physica C 468, 2046-2049 (2008)

16. Bashkirov, Y., Fleishman, L., Patsayeva, T., Sobolev, A., Vdovin, A.: Current-limiting reactor based on high-Tc superconductors. IEEE Trans. Magnet. 27, 1089-1092 (1991)

17. Paul, W., Lakner, M., Rhyner, J., Unternährer, P., Baumann, T., Chen, M., Windnhorn, L., Guérig, A.: Test of 1.2 MVA high-Tc superconducting fault current limiter. Superconductor Science and Technology 10, 914-918 (1997)

18. Elschner, S., Breuer, F., Walter, H., Stemmle, M., Bock, J.: HTS Components for High Voltage Resistive Current Limiters Based on a Magnetic Field Triggered Concept. IEEE Trans. App. Superconductivity 17, 1772-1775 (2007) 